\title{
Factors associated with the outcome of transforaminal epidural steroid injections
}

\author{
Department of Anesthesiology and Pain Medicine, Dong San Medical Center, Keimyung University School of Medicine; \\ *Mom Pyun Han Hyu Clinic, Daegu, Korea
}

Tae Kyu Park, M.D.*, Ji Hee Hong, M.D., Jin Mo Kim, M.D., Jeong In Bae, M.D., Ae Ra Kim, M.D., Han-Min Lee, M.D., and Yong-Cheol Lee, M.D.

Background: This study was undertaken to assaythe effectiveness of transforaminal epidural steroid injections (TFESIs) for sciatica and to identify potential predictors related to treatment outcome.

Methods: TFESIs were performed in 150 patients between August 2006 and March 2007. Seventy-five patients (35 women, 40 men; mean age, 59.1 years), who met the inclusion criteria, were studied. Therapeutic effects were evaluated twoweeks after injection. The following potential outcome predictors analyzed were as follows: one level vs. two level injection, Beck depression inventory score $(<13,14-19,>20)$, Beck anxiety inventory score $(<7,8-15,>16)$, cause of radiculopathy (spinal stenosis vs. herniated disk), gender, duration of radiculopathy ( $<6$ months, 6-12 months, $>6$ months), and Oswestry disability index score $(<30,31-60,>60)$. The relationships between possible outcome predictors and therapeutic effects were evaluated.

Results: Forty-nine of the 75 patients $(65.3 \%)$ had a satisfactory result two weeks after TFESIs. Of these, twenty-four of the 46 patients $(52.2 \%)$ were treated by a one level injection and $25(89.3 \%)$ of the 29 patients were treated by a two level injection. This outcome was statistically significant $(\mathrm{P}<0.01)$. None of the other potential outcome predictors showed any statistical difference.

Conclusions: TFESI is recommended as an effective method of managing radiculopathy. Two-level injectionsmay result in a better outcome than a one-level injection. (Korean J Anesthesiol 2008; 55: 298 304)

Key Words: outcome predictors, transforaminal epidural steroid injections, two level.

\section{INTRODUCTION}

Sciatica is a common, benign problem with a prevalence estimated at approximately $13-40 \%$ among general population. ${ }^{1)}$ Based on recent understanding of pain generation in sciatica, it is believed that the development of severe sciatica depends not only on mechanical compression but also on concomitant chemical irritation of the nerve root caused by a disk material. $^{2-4)}$ Therefore, local application of corticosteroids to the compressed and inflamed nerve root is a reasonable treatment option. Percutaneous injection therapies, including transforaminal

Received : February 29, 2008

Corresponding to : Ji Hee Hong, Department of Anesthesiology and Pain Medicine, Dong San Medical Center, Keimyung University School of Medicine, 194, Dongsan-dong, Jung-gu, Daegu 700-712, Korea. Tel: 82-53-250-7542, Fax: 82-53-250-7240

E-mail: pain1004@dsmc.or.kr

4th WIP (World Institue of Pain), 2007, budapest, Hungary epidural steroid injection (TFESI), are being used with increasing frequency to treat sciatica resulting from a herniated intervertebral disk (HIVD) or degenerative lumbar spinal stenosis. ${ }^{1,5,6)}$

Three principal avenues are available for delivering medication into the epidural space, viz. the transforaminal, interlaminar and caudal routes. TFESI performed under fluoroscopic guidance has become the most specific and effective route of administration. ${ }^{6,7)}$ The advantages of the transforaminal approach are that corticosteroid preparations can be injected closer to the probable source of irritated nerve root $^{6)}$ and that it results in better ventral epidural spreading than the interlaminar approach. ${ }^{7)}$

It is very important to clarify the predictor related to outcome and Jeong et al. ${ }^{8)}$ suggested a pre-ganglionic approach at the level of the supra-adjacent intervertebral disc level, Lee et al. ${ }^{9)}$ suggested an extraepineural injection pattern as a potential better outcome predictor.

The objectives of this study were to verify the potential pre- 
dictors of TFESI outcome. We will describe conventional TFESI as one level method and pregan glionic TFESI two level method.

\section{MATERIALS AND METHODS}

\section{Patients}

A single pain specialist retrospectively reviewed the medical records of 150 patients who received one-level or two-level TFESI between July 2006 and March 2007 for lumbar radicular pain. We obtained institutional review board and patients approval. From among these patients, we enrolled those who met the following inclusion criteria: 1) symptoms of lumbar radiculopathy 2) no prior therapeutic transforaminal epidural steroid injection 3) no prior lumbar spine surgery 4) radicular pain consistent with the L5 or S1 dermatomal distribution, and clear identification of L5 or S1 root lesion by MR imaging or CT 5) availability of follow-up data two weeks after injection. In the one-level TFESI method, TFESI was performed at the L5-S1 or S1 foramen by the conventional approach (Fig. 1A). In the two-level TFESI method, TFESI was performed by the conventional method at the affected nerve root level, and a supplementary TFESI was performed at the supra-adjacent intervertebral disc level by the pre-ganglionic approach (Fig. 1B). A total of 75 patients who fulfilled the above inclusion criteria were enrolled. All patients had undergone CT or MR imaging before the procedure, and patients were informed of the potential risks associated with the procedure and the use of steroids and provided informed consent.
A
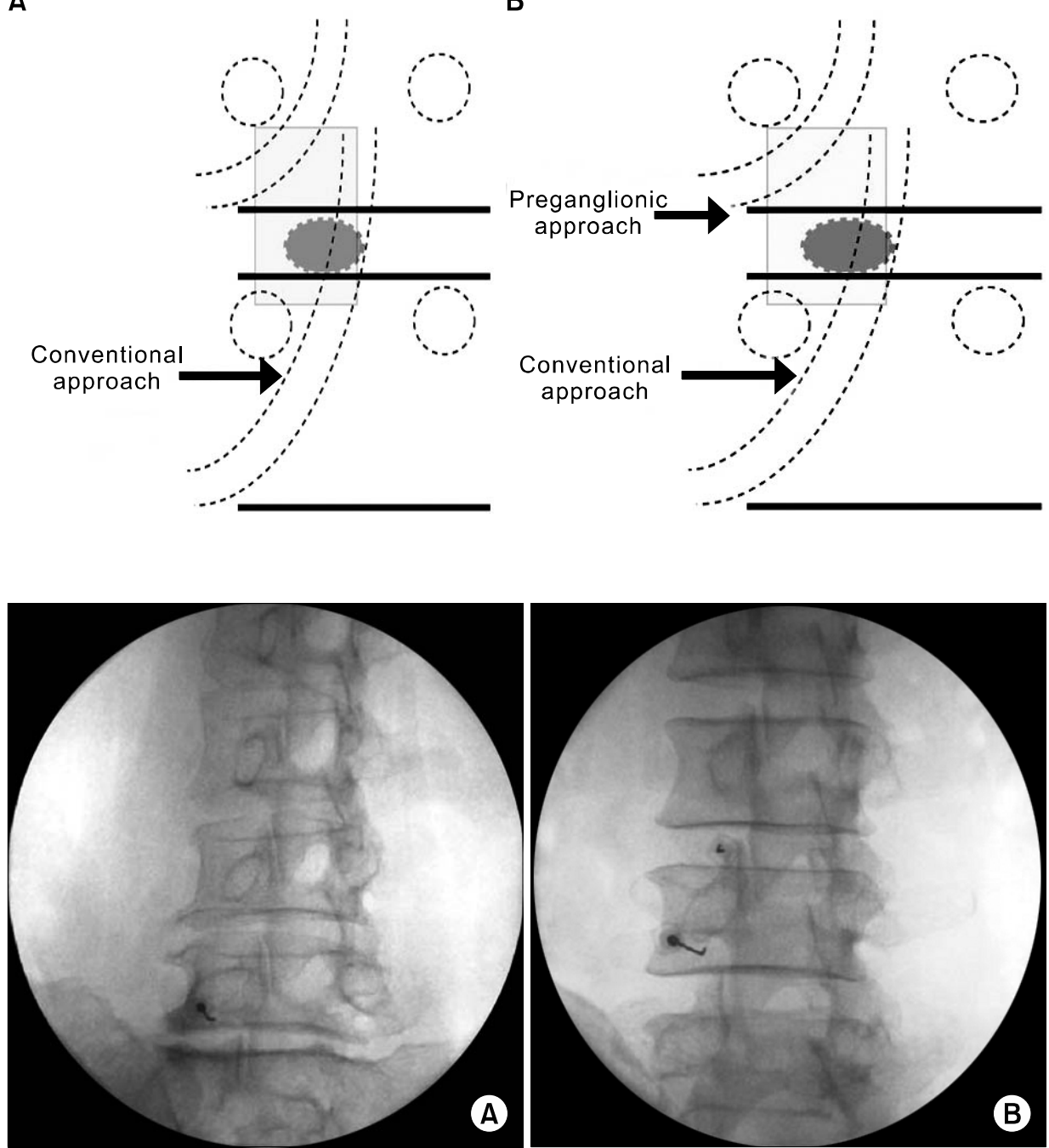

Conventional approach

B

Preganglionic approach

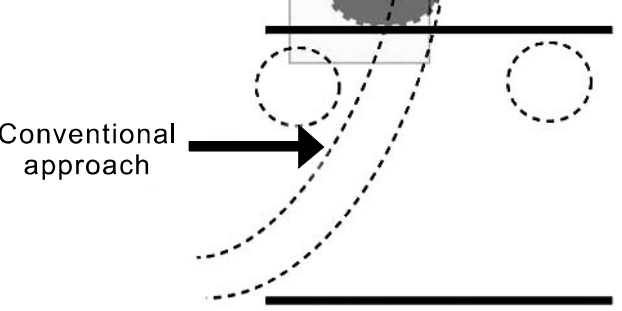

Fig. 1. (A) Schematic diagram showing conventional approach. (B) Schematic diagram showing supplementary preganglionic approach.
Fig. 2. (A) Olique view of transforaminal epidural steroid injection showing conventional approach at the L5-S1 level. (B) Oblique view of transforaminal epidural steroid injection showing supplementary preganglionic approach at the L3-4 and L4-5 level. 


\section{Techniques}

All therapeutic TFESIs were conducted under C-arm guidance by a pain specialist experienced in performing spinal interventions. All the injections were performed as outpatient procedures, and informed consent was obtained from each patient.

One-level TFESIs were performed by a conventional method. With a patient lying prone, the $\mathrm{C}$-arm was tilted with craniocaudal angulation to align the inferior end plate of the target vertebra. Then, an oblique view was obtained by rotating the $\mathrm{C}$-arm until the superior articular process was at the lateral third on the superior end plate (Fig. 2A). In the two-level TFESI group, a conventional TFESI was performed at the L5-S1 or L4-5 level with the same method used for the one-level group, and a supplementary pre-ganglionic approach was done at the supra-adjacent intervertebral disc level. For the pre-ganglionic approach, we targeted injections just lateral to the superior articular process and parallel to the inferior end plate of the vertebral body on the oblique view (Fig. 2B).

The goal of positioning was to get a perpendicular needle tract toward the classic injection site and underneath the pedicle in the so called "safe triangle". ${ }^{7)}$ With C-arm guidance, a 9 $\mathrm{cm}, 20$ gauge epidural needle was advanced into the safe triangle. Two $\mathrm{ml}$ of contrast material (Omnipaque 300, GE Healthcare, Little Chalfont, Buckinghamshire, UK) was injected to confirm epidural spread and to avoid intravascular, intrathecal or soft tissue infiltration (Fig. 3A, 4A). Patients' epidurograms were carefully reviewed. In the one-level TFESI group, $4 \mathrm{ml}$ of injectate was slowly injected, which consisted
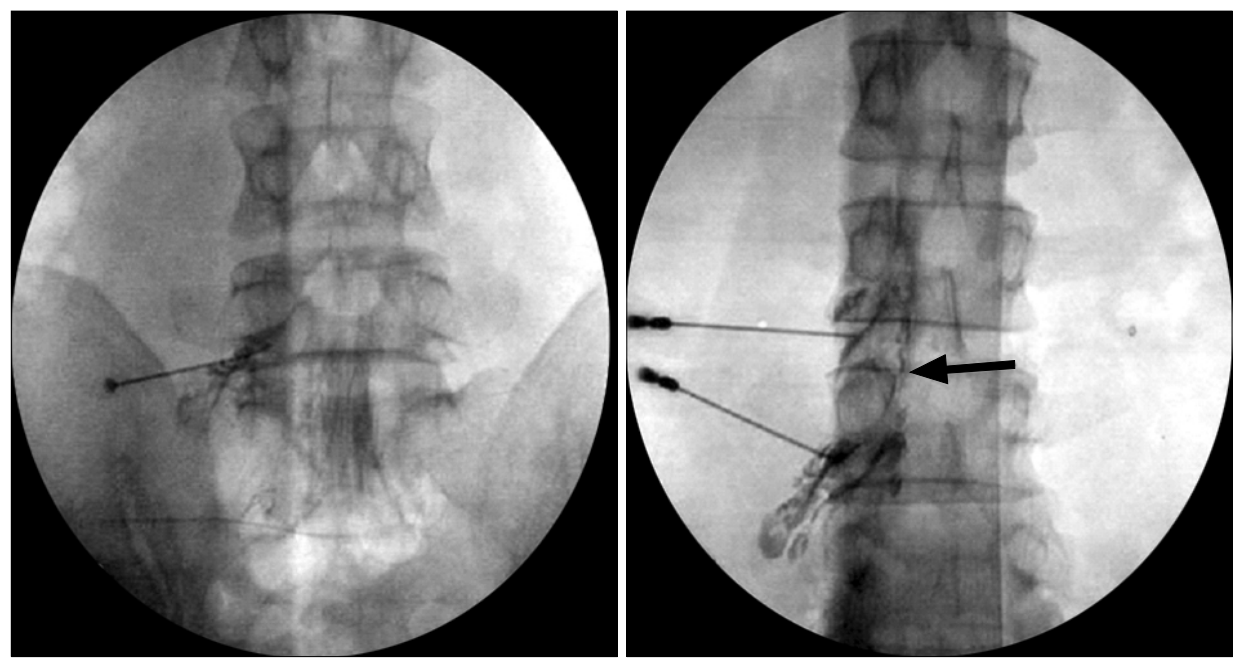

Fig. 3. (A) Anteroposterior view of transforaminal epidural steroid injection showing conventional approach at the L5-S1 level. (B) Anteroposterior view of transforaminal epidural steroid injection showing supplementary preganglionic approach at the L3-4 and L4-5 level. Arrow indicates preganglionic flow.
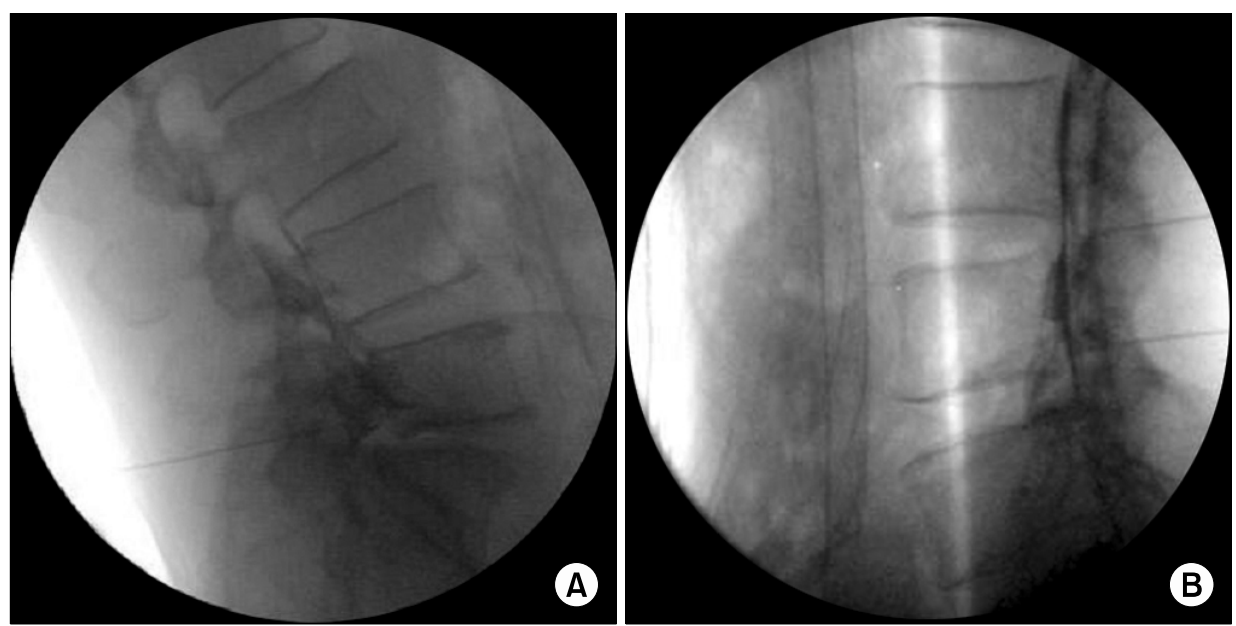

Fig. 4. (A) Lateral view of transforaminal epidural steroid injection showing conventional approach at the L5-S1 level. (B) Lateral view of transforaminal epidural steroid injection showing supplementary preganglionic approach at the L3-4 and L4-5 level. 
of $1 \mathrm{ml} \mathrm{2 \%}$ mepivacaine hydrochloride, $1 \mathrm{ml}$ of $40 \mathrm{mg}$ triamcinolone acetonide suspension, and $2 \mathrm{ml}$ of contrast material.

The final needle position was at the inferior aspect of the supra-adjacent neural foramen, with the bevel immediately dorsal to the annulus of the interverterbral disc and the posterior longitudinal ligament. Four $\mathrm{ml}$ of injectate with the same composition as the one-level group was divided into two $2 \mathrm{ml}$ aliquots, and $2 \mathrm{ml}$ of injectate was administered at each level (Fig. 3B, 4B). There was no intrathecal or intravascular injection.

\section{Review of clinical data}

Follow-up interviews were conducted at the hospital two weeks after TFESI by an observer blinded to the technique used at the time of injection. Pain severity was assessed by a self-administered visual analogue scale (VAS, range $0-100$ $\mathrm{mm}$ ) both before the procedure and at the two-week follow-up interview. Physical disability was measured by the Oswestry disability index (ODI, $0-100 \%$ ), where $0 \%$ represents no disability and $100 \%$ extreme disability. ${ }^{10}$ Depression and anxiety were gauged with the 21-item Beck depression inventory (BDI, $0-63$ ) and the Beck anxiety inventory (BAI, 0-63) respectively. ${ }^{11)}$ The ODI, BDI, and BAI were assessed both before the procedure and at the two-week follow-up interview. We also adopted a 5-point patient outcome scale for post-treatment pain assessments as follows: 0 (aggravated), 1 (stationary), 2 (improved), 3 (much improved), and 4 (no residual symptoms). We asked patients who visited the hospital two weeks after TFESI about their pain intensity, then compared them with those of pre-treatment according to the 5-point patient outcome scale. An effective treatment was defined as a score of 3 or 4 and a VAS score reduction of more than $50 \%$ after TFESI.

Potential predictors of the effectiveness of treatment with TFESI that were postulated included one-level versus two-level injection, BDI score $(0-13,14-19,>19)$, BAI score $(0-7$, $8-15,>15$ ), cause of radiculopathy (spinal stenosis vs. HIVD), gender, duration of symptoms (0-6 month, 6-12 months, $>12$ months), and ODI $(0-30 \%, 31-60 \%,>60 \%)$.

\section{Statistical Analysis}

All variables were tested for normal distribution using the Kolmogorov-Smirnov test. Normally distributed variables are expressed as mean $\pm \mathrm{SD}$. The chi-square test was used to examine the association of therapeutic outcome and the seven variables (TFESI approach, BDI score, BAI score, cause of radiculopathy, gender, duration of symptom, ODI). For the seven variables evaluated, we also performed logistic regression

Table 1. Possible Outcome Predictors of TFESI and its Effectiveness

\begin{tabular}{|c|c|c|c|c|}
\hline & & Effective & Ineffective & Total \\
\hline \multirow{2}{*}{ TFESI approach } & 1 level & $24(52.1 \%)$ & $22(47.8 \%)$ & 46 \\
\hline & 2 level & $25(86.2 \%)^{*}$ & $4(13.7 \%)$ & 29 \\
\hline \multirow[t]{3}{*}{ BDI score } & $0-13$ & $17(56.6 \%)$ & $13(43.3 \%)$ & 30 \\
\hline & $14-19$ & $21(75.0 \%)$ & $7(25.0 \%)$ & 28 \\
\hline & $>19$ & $11(64.7 \%)$ & $6(35.2 \%)$ & 17 \\
\hline \multirow[t]{3}{*}{ BAI score } & $0-7$ & $27(69.2 \%)$ & $9(30.7 \%)$ & 36 \\
\hline & $8-15$ & $10(55.5 \%)$ & $8(44.4 \%)$ & 18 \\
\hline & $>15$ & $12(57.1 \%)$ & $9(42.8 \%)$ & 21 \\
\hline \multirow[t]{2}{*}{ Cause of radiculopathy } & Spinal stenosis & $33(64.7 \%)$ & $18(35.2 \%)$ & 51 \\
\hline & HIVD & $16(66.6 \%)$ & $8(33.3 \%)$ & 24 \\
\hline \multirow[t]{2}{*}{ Gender } & Male & $29(72.5 \%)$ & $11(27.4 \%)$ & 40 \\
\hline & Female & $20(57.1 \%)$ & $15(42.8 \%)$ & 35 \\
\hline \multirow[t]{3}{*}{ Duration of symptoms (month) } & $0-6$ & $36(65.4 \%)$ & $19(34.5 \%)$ & 55 \\
\hline & $6-12$ & $7(63.6 \%)$ & $4(36.3 \%)$ & 11 \\
\hline & $>12$ & $6(66.6 \%)$ & $3(33.3 \%)$ & 9 \\
\hline \multirow[t]{3}{*}{ ODI } & $0-30$ & $11(61.1 \%)$ & $7(38.8 \%)$ & 18 \\
\hline & $31-60$ & $33(73.3 \%)$ & $12(26.6 \%)$ & 45 \\
\hline & $>60$ & $5(41.6 \%)$ & $7(58.3 \%)$ & 12 \\
\hline
\end{tabular}

Values are number of patients. TFESI: transforaminal epidural steroid injection, HIVD: herniated intervertebral disc, BDI: Beck depression inventory, BAI: Beck anxiety inventory, ODI: Oswestry disability index. *: $\mathrm{P}<0.01$ (1-level vs. 2-level). 
Table 2. Logistic Regression Analysis for the Possible Predictors of Outcome for the Effectiveness of TFESI

\begin{tabular}{lcccccc}
\hline & B & SE & Wald & df & Sig & Exp(B) \\
\hline One level vs. two level approach & 1.746 & 0.614 & 8.080 & 1 & 0.004 & 5.729 \\
BDI score & -0.473 & 0.346 & 1.871 & 1 & 0.171 & 0.623 \\
BAI score & 0.471 & 0.315 & 2.232 & 1 & 0.135 & 1.601 \\
Cause of radiculopathy & -0.237 & 0.659 & 0.129 & 1 & 0.719 & 0.789 \\
Gender & 0.649 & 0.564 & 1.323 & 1 & 0.250 & 1.913 \\
Duration of symptoms & -0.251 & 0.405 & 0.383 & 1 & 0.536 & 0.778 \\
ODI & 0.314 & 0.438 & 0.515 & 1 & 0.473 & 1.369 \\
\hline
\end{tabular}

TFESI: transforaminal epidural steroid injection, HIVD: herniated intervertebral disc, BDI: Beck depression inventory, BAI: Beck anxiety inventory, ODI: Oswestry disability index. B: regression coefficient, SE: standard error, Wald: Wald statistics, df: degree of freedom, Exp(B): odds ratio.

analysis to reveal any correlations between therapeutic effectiveness and possible outcome predictors. To compare the mean values of ODI, BDI, BAI, and VAS between groups and before and two weeks after the TFESI procedure, independent and paired t-tests were performed (SPSS v. 12, Chicago, IL).

\section{RESULTS}

The study subjects included 35 women and 40 men with a mean age of 62.8 years (age range, 19-91; SD, 14.4). The mean age was 59.1 years $(\mathrm{SD}, 12.0)$ in the one-level TFESI group and 56.6 years $(\mathrm{SD}, 17.1)$ in the two-level TFESI group. The difference in mean age was not statistically significant.

One-level TFESI was performed in 46 patients $(62.2 \%)$ and was effective in 24 (52.2\% of recipients), and two-level TFESI was performed in 29 patients (38.6\%) and was effective for 25 (89.3\% of recipients) $(\mathrm{P}<0.01)$. The difference in the two rates of effective treatment was significant $(\mathrm{P}=0.004$; odds ratio $=5.729)$. In contrast, BDI score, BAI score, cause of radiculopathy, gender, duration of symptoms, and ODI were not found to be statistically related to the effectiveness of TFESI (Table 1,2).

When ages and sex distribution were compared, there was no difference in effectiveness between one level and two level method. However, as a cause of radiculopathy, lumbar HIVD showed significant effectiveness than spinal stenosis in a two level group.

In one-level group, 20 patients showed a rostral epidural spread pattern and 26 patients had a nonrostral pattern. In two-level group, a rostral epidural spread pattern was found in all patients (Fig. 2A-4A, Fig. 2B-4B). In the one-level TFESI group, 34 patients $(73.9 \%)$ had spinal stenosis and 12 patients (26.0\%) had HIVD, while in the two-level TFESI
Table 3. ODI, BDI, BAI, VAS Scores

\begin{tabular}{lcc}
\hline & $\begin{array}{c}\text { Group one-level } \\
(\mathrm{n}=46)\end{array}$ & $\begin{array}{c}\text { Group two-level } \\
(\mathrm{n}=29)\end{array}$ \\
\hline ODI before & $44.8 \pm 14.6$ & $40.7 \pm 14.8$ \\
ODI after & $33.1 \pm 14.1^{*}$ & $26.6 \pm 14.0^{*}$ \\
BDI before & $16.2 \pm 8.5$ & $17.5 \pm 9.2$ \\
BDI after & $14.1 \pm 5.5$ & $15.5 \pm 6.5$ \\
BAI before & $10.1 \pm 8.2$ & $11.9 \pm 10.0$ \\
BAI after & $9.6 \pm 3.2$ & $10.0 \pm 2.0$ \\
VAS before & $69.5 \pm 9.5$ & $66.4 \pm 12.6$ \\
VAS after & $33.4 \pm 18.7^{*}$ & $24.6 \pm 14.0^{*}$ \\
\hline
\end{tabular}

Values are mean \pm SD. ODI: Oswestry disability index $(0-100 \%)$, BDI: Beck depression inventory $(0-63)$, BAI: Beck anxiety inventory $(0-63)$, VAS: Visual analogue scale $(0-100 \mathrm{~mm})$, before: before the procedure, after: 2 wk follow up after the procedure. *: $\mathrm{P}<0.01$ within group (mean $\pm \mathrm{SD}$ ).

group 17 patients $(58.6 \%)$ had spinal stenosis and 12 patients (41.3\%) had HIVD. The radiculopathy was acute (0-6 months) or subacute $(6-12$ months) in 32 patients $(69.5 \%)$ and chronic $(>12$ months) in 14 patients $(30.4 \%)$ in the one-level TFESI group, and in the two-level TFESI group, radiculopathy was acute or subacute in 25 patients $(86.2 \%)$ and chronic in 4 patients (13.7\%).

The mean BDI score was 16.2 in the one-level TFESI group, and 17.5 in the two-level TFESI group. TFESI was performed in the one-level TFESI group at L4-5 in 14 patients and at L5-S1 in 32 patients. In the two-level TFESI group, TFESI was performed at L3-4 and L4-5 in 6 patients, and L4-5 and L5-S1 in 23 patients.

ODI and VAS were significantly decreased within groups after treatment (before vs. $2 \mathrm{wk}, \mathrm{P}<0.01$ ) but there were no significant differences between groups. BDI and BAI were not significantly different either within groups or between groups (Table 3). 


\section{DISCUSSION}

Several causes of nerve root irritation have been previously reported, including direct chemical effects resulting from leakage of substances such as phopholipase A2 from the intervertebral disk; mechanical compression; indirect effects such as vascular compression; and a combination of pathogenetic factors. $^{2-4)}$ The nucleus pulposus possesses inflammatogenic properties, as indicated by leukotaxis and an increase in vascular permeability, ${ }^{4}$ and it has been reported that autologous nucleus pulposus injected into the lumbar epidural space of dogs causes a chemical or immunologic inflammation of the neural sac. ${ }^{12)}$ Olmarker et al. ${ }^{4)}$ observed that epidural application of autologous nucleus pulposus in pigs, without mechanical nerve root compression, induced a pronounced reduction in nerve conduction velocity in the nerve roots of the cauda equina. This observation suggested a mechanism that nucleus pulposus can affect directly on nerve fiber structures and function via biochemical effects.

The anti-inflammatory properties of corticosteroids are well known, and they are often selected for local application to relieve reversible inflammatory changes or processes, such as vascular congestion related to mechanical obstruction. ${ }^{1,5,6)}$ In an experimental animal study, it was reported that the effect of an ESI was related to the inhibition of phopholipase A2 activity. ${ }^{13)}$ Therefore, in the treatment of patients with intractable sciatica, non-operative measures should be considered, and the use of therapeutic TFESI to deliver corticosteroid locally appears to be a reasonable approach.

The transforaminal approach to the epidural space has gained acceptance because of its improved specificity and its utility as both a diagnostic and a therapeutic intervention. Delivery of a therapeutic injectate transforaminally at the involved nerve root maximizes steroid concentration at the site of pathology and minimizes the dilution effect within the epidural space. ${ }^{5-7)}$

In our study, follow up was conducted two weeks after TFESI, which was a time consistent with what has been proposed in the literature for follow up. And it was based on the duration of the therapeutic effect of corticosteroids. ${ }^{1,13)}$ Most previous investigations of the duration of pain relief after spinal steroid injections had showed a short or a little longer time of pain relief after spinal steroid injection that is three months at most. Although the findings of most study indicate a markedly declining effect after three months, ${ }^{14)}$ there is also evidence of a potential long-term effect, ${ }^{15)}$ which was not investigated in our study.

We found that TFESI was effective in $65.3 \%$ of patients with disk herniation or spinal stenosis. We could not find any statistical difference in therapeutic outcome between spinal stenosis and HIVD, and several other studies have reported a similar result. ${ }^{11,16)}$ The BDI is one of the most widely used self-reporting instruments for measuring the severity of depression, which was developed to assess the type and severity of depression based on symptoms. One of the advantages of the BDI is its usefulness in screening for depression in general populations in addition to its utility for measuring the severity of depression among psychiatric patients. ${ }^{17)}$ Although an association between low back pain and depression has been reported previously, ${ }^{18-20)}$ we did not find any association between therapeutic outcome and either BDI or BAI.

We observed an improved mean score for ODI and VAS at the two-week follow up point in both groups, but there were no significant differences found in either BDI or BAI. Acherman and $\mathrm{Ahmad}^{21)}$ reported that they could find significantly decreased scores on both the Oswestry low back pain scale and BDI at two weeks after the last lumbar epidural injection. It is thought that as we examined the BDI or BAI score only two weeks after first injection, it is considered too short time interval to find any significant changes in BDI or BAI score. Acherman and $\mathrm{Ahmad}^{21)}$ also examined Oswestry low back pain scale and BDI at two weeks after last injection, however they had enough time than our study because they examined scores after performing injections at least two or three times.

Our data showed a better clinical outcome in two-level than in one-level TFESI. Lee et $\mathrm{al}^{22}$ reported that pre-ganglionic TFESI had a better therapeutic effect on radiculopathy than the conventional method.

Why is two-level injection more effective than one level injection? The explanation may be that two-level injection has more chances to spread into the more effective sites than one-level injection. Ideally, if there is no stenosis and the needle tip is appropriately positioned, the injectate may spread rostrally to the epidural portion of the nerve root. However, for most cases of lumbosacral radiculopathy that are secondary to stenosis or a herniated disc, the site of impingement lies at the level of the supra-adjacent intervertebral disc, which is rostral to the conventional lumbar TFESI bevel position. In our study, in one-level group 20 patients showed a rostral epidural spread pattern and 26 patients exhibited a nonrostral pattern. 
However, in two-level group we added a supplementary preganglinoic TFESI at the supra-adjacent disc level, therefore achieving a rostral epidural spread pattern in all patients. Moreover, the advantage of pre-ganglionic TFESI is that the injected medication distributes predominantly in the epidural space at the disc level and does not excessively spread along the spinal nerve, which is not inflamed or mechanically compressed. Thus, if after performing an injection to the most affected nerve root and no rostral spreading is seen, adding a supplementary injection to the upper root level may be a more effective method to deliver injectate to the target site.

The present study is a retrospective analysis of patients with sciatica treated using transforaminal epidural steroid injection, and its retrospective design is a limitation of the study. We also included patients with both spinal stenosis and lumbar HIVD, which are distinctly different disease entities. Another limitation of our study is that we observed outcomes in only a short interval, and other factors that could have influenced the therapeutic effect were not considered. And it was not a complete assessment as we did not include the BDI, BAI, and ODI for defining the effective treatment. Finally, direct comparison between rostral and nonrostral spread was difficult because we included patients with both rostral and nonrostral spread patterns in one-level group.

In conclusion, the results of our study suggest that TFESI is an effective method for managing sciatica. The two-level injection may bring a better outcome in treating sciatica due to HIVD or spinal stenosis using TFESI.

\section{REFERENCES}

1. Stafford MA, Peng P, Hill DA: Sciatica: A review of history, epidermiology, pathogenesis, and the role of epidural steroid injection in management. Br J Anaesth 2007; 99: 461-73.

2. Nygaard OP, Mellgren SI, Osterud B: The inflammatory properties of contained and noncontained lumbar disc herniation. Spine 1997; 22: $2484-8$.

3. Franson RC, Saal JS, Saal JA: Human disc phopholipase A2 is inflammatory. Spine 1992; 17(6 Suppl): 129-32.

4. Olmarker K, Blomquist J, Stromberg J, Nannmark U, Thomsen P, Rydevik B: Inflammatogenic properties of nucleus pulposus. Spine 1995; 20: 665-9.

5. Thomas E, Cyteval C, Abiad L, Picot MC, Taourel P, Blotman F: Efficacy of transforaminal versus interspinous corticosteroid injection in discal radiculalgia-a prospective, randomized, double-blind study. Clin Rheumatol 2003; 22: 299-304.

6. Manchikanti L: Transforaminal lumbar epidural steroid injections.
A focused review. Pain Physician 2000; 3: 374-98.

7. Botwin K, Natalicchio J, Brown LA: Epidurography contrast patterns with fluoroscopic guided lumbar trasnforaminal epidural injections. A prospective evaluation. Pain Physician 2004; 7: 211-5.

8. Jeong HS, Lee JW, Kim SH, Myung JS, Kim JH, Kang HS: Effectiveness of transforaminal epidural steroid injection by using a preganglionic approach: a prospective randomized controlled study. Radiol 2007; 245: 584-90.

9. Lee JW, Kim SH, Lee IS, Choi JA, Choi JY, Hong SH, et al: Therapeutic effect and outcome predictors of sciatica treated using transforaminal epidural steroid injection. Am J Roentgenol 2006; 187: $1427-31$.

10. Haro H, Maekawa S, Hamada Y: Prospective analysis of clinical evaluation and self-assessment by patients after decompression surgery for degenerative lumbar canal stenosis. Spine J 2008; 8: 380-4.

11. Lykke J, Hesse M, Austin SF, Oestrich I: Validity of the BPRS, the BDI and the BAI in dual diagnosis patients. Addict Behav 2008; 33: 292-300.

12. McCarron RF, Wimpee MW, Hudkins PG, Laros GS: The inflammatory effect of nucleus: a possible element in the pathogenesis of low back pain. Spine 1987; 12: 760-4.

13. Lee HM, Weinstein JN, Meller ST, Hayashi N, Spratt KF, Gebhart GF: The role of steroids and their effects on phopholipase A2: an animal model of radiculopathy. Spine 1998; 23: 1191-6.

14. Carette S, Leclaire R, Marcoux S, Morin F, Blaise GA, St-Pierre A, et al: Epidural corticosteroid injections for sciatica due to herniated nucleus pulposus. N Engl J Med 1997; 336: 1634-40.

15. Delport EG, Cucuzzella AR, Marley JK, Pruitt CM, Fisher JR: Treatment of lumbar spinal stenosis with epidural steroid injections: A retrospective outcome study. Arch Phys Med Rehabil 2004; 85: 479-84.

16. Lee IS, Kim SH, Lee JW, Hong SH, Choi JY, Kang HS, et al: Comparison of the temporary diagnostic relief of transforaminal epidural steroid injection approaches: Conventional versus poterolateral technique. Am J Neuroradiol 2007; 28: 204-8.

17. Jo SA, Park MH, Jo I, Ryu SH, Han C: Usefulness of Beck depression inventory (BDI) in the Korean elderly population. Int $\mathrm{J}$ Geriatr Psychiatry 2007; 22: 218-23.

18. Carragee EJ: Psychologic and functional profiles in select subjects with low back pain. Spine J 2001; 1: 198-204.

19. Croft PR, Papageorgiou AC, Ferry S, Thomas E, Jayson MI, Silman AJ: Psychologic distress and low back pain: Evidence from a prospective study in the general population. Spine 1995; 20: 2731-7.

20. Hansen F, Biering-Sorensen F, Schroll M: Minnesota Multiphasic Personality Inventory profiles in person with or without low back pain: A 20-year follow-up study. Spine 1995; 20: 2716-20.

21. Ackerman WE 3rd, Ahmad M: The efficacy of lumbar epidural steroid injections in patients with lumbar disc herniations. Anesth Analg 2007; 104: 1217-22.

22. Lee JW, Kim SH, Choi JY, Yeom JS, Kim KJ, Chung SK, et al: Transforaminal epidural steroid injection for lumbosacral radiculopathy: Preganglionic versus conventional approach. Korean J Radiol 2006; 7: 139-144. 